\title{
Waking EEG functional connectivity in middle-aged and older adults with obstructive sleep apnea
}

Maxime Fortin ${ }^{\mathrm{a}, \mathrm{b}}$, Jean-Marc Lina ${ }^{\mathrm{a}, \mathrm{c}}$, Marie-Ève Desjardinss,d, Katia Gagnon ${ }^{\mathrm{a}, \mathrm{e}}$, Andrée-Ann Baril $^{\mathrm{a}, \mathrm{e}}$, Julie Carrier ${ }^{\mathrm{a}, \mathrm{d}} \&$ Nadia Gosselin ${ }^{\mathrm{a}, \mathrm{d}}$.

a. Center for Advanced Research in Sleep Medicine, CIUSSS du Nord de l'Île de Montréal, Hôpital du SacréCoeur de Montréal, 5400 boulevard Gouin Ouest, Montréal, Québec, Canada, H4J 1C5.

b. Département de Psychologie, Université du Québec à Montréal, Pavillon Adrien-Pinard, C.P. 8888 succursale Centre-ville, Montréal, Québec, Canada, H3C 3P8.

c. Département de Génie Électrique, École de technologie supérieure, 1100 Notre-Dame Ouest, Montréal, Canada, H3C 1K3.

d. Département de Psychologie, Université de Montréal, Pavillon Marie-Victorin, C. P. 6128, succursale Centre-ville, Montréal, Québec, Canada, H3C 3J7.

e. Département de Psychiatrie, Faculté de médecine, Université de Montréal, Pavillon Roger-Gaudry, C.P. 6128, succursale Centre-ville, Montréal, Québec, Canada, H3C 3J7.

\section{E-mails}

Maxime Fortin: fortin.maxime.7@ courrier.uqam.ca

Jean-Marc Lina: Jean-Marc.Lina@etsmtl.ca

Marie-Ève Desjardins: marie-eve.desjardins.2@umontreal.ca

Katia Gagnon: katia.gagnon.ceams@gmail.com

Andrée-Ann Baril: baril.andreeann@gmail.com

Julie Carrier: julie.carrier.1@ umontreal.ca

Nadia Gosselin: nadia.gosselin@umontreal.ca

Running head: EEG connectivity in OSA

Total word count: 4311 words (excluding the abstract, acknowledgments and references)

Declaration of interest: Mr. Fortin reports other from Conseil de recherches en sciences naturelles et en génie du Canada; Dr. Desjardins reports other from Fonds de la recherche du Québec, nature et technologies; Dr. Gagnon reports personal fees from Hôpital du Sacré-Cœur de Montréal, personal fees from Fonds de la recherche du Québec - Nature et technologie; Dr. Baril reports other from Fonds de la recherche du Québec - Santé, other from Canadian Institutes of Health Research; Dr. Carrier reports grants from Canopy Health, grants from Merck, grants from Rana, grants from Philipps-Respironics, outside the submitted work, during the conduct of the study. Dr. Gosselin reports a grant and a salary award from the Fonds de la recherche du Québec - Santé and a grant from the Canadian Institutes of Health Research.

\section{Corresponding author:}

Nadia Gosselin, $\mathrm{PhD}$

Center for Advanced Research in Sleep Medicine

Hôpital du Sacré-Cœur de Montréal

5400 Gouin Blvd. West, Room J-5135

Montreal, Quebec, H4J 1C5, Canada

Tel.: 514-338-2222, ext. 7717; Fax: 514-338-3893

Email: nadia.gosselin@umontreal.ca 


\begin{abstract}
Objectives: The present study aimed at investigating changes in waking electroencephalography (EEG), most specifically regarding spectral power and functional connectivity, in middle-aged and older adults with OSA. We also explored whether changes in spectral power or functional connectivity are associated with polysomnographic characteristics and/or neuropsychological performance.
\end{abstract}

Methods: 19 OSA subjects (apnea-hypopnea index $\geq 20$, age: $63.6 \pm 6.4$ ) and 22 controls (apneahypopnea index $\leq 10$, age: $63.6 \pm 6.7$ ) underwent a full night of in-laboratory polysomnography followed by a waking EEG and a neuropsychological assessment. Waking EEG spectral power and imaginary coherence were compared between groups for all EEG frequency bands and scalp regions. Correlation analyses were performed between selected waking EEG variables, polysomnographic parameters and neuropsychological performance.

Results: No group difference was observed for EEG spectral power for any frequency band. Regarding the imaginary coherence, when compared to controls, OSA subjects showed decreased EEG connectivity between frontal and temporal regions in theta and alpha bands as well as increased connectivity between frontal and parietal regions in delta and beta 1 bands. In the OSA group, these changes in connectivity correlated with lower sleep efficiency, lower total sleep time and higher apnea-hypopnea index. No relationship was found with neuropsychological performance.

Conclusions: Contrary to spectral power, imaginary coherence was sensitive enough to detect changes in brain function in middle-aged and older subjects with OSA when compared to controls. Whether these changes in cerebral connectivity predict cognitive decline needs to be investigated longitudinally. 
Keywords: Sleep-related breathing disorder; quantitative electroencephalography; wakefulness; functional connectivity; imaginary coherency.

Statement of significance: This research offers new insight about how using EEG functional connectivity could be a sensitive tool to detect effects of OSA on brain function. Using the imaginary part of the coherency with a nonparametric design, we evaluated functional interactions between different brain regions, and we detected subtle changes in brain function in middle-aged and older adults with OSA. Increases as well as decreases in functional brain connectivity were found in OSA and they were associated with a poorer sleep quality on the polysomnography. In contrast to previous studies having found a general slowing of the EEG in OSA, no changes were found using EEG spectral power in this study, suggesting that functional connectivity is more sensitive to the effects of OSA than EEG spectral analysis. 


\section{Introduction}

Obstructive sleep apnea (OSA) can affect up to $50 \%$ of adults after the age of 65 [1]. In OSA, repetitive arousals and intermittent hypoxemia associated with apneas and hypopneas can have deleterious effects on brain health [2]. Systematic reviews and meta-analyses showed that OSA impacts attention and vigilance, episodic memory, executive functions, information processing speed, as well as visuospatial and constructional abilities [3-6]. Whether these cognitive dysfunctions could lead to abnormal cognitive aging and dementia is still the focus of important research efforts, but one meta-analysis reported that untreated OSA is associated with a $26 \%$ increase in the risk of developing cognitive decline or dementia [7]. It is therefore important to verify whether middle-aged and older adults with untreated OSA present subtle abnormalities in their cerebral functioning and whether these changes are associated with cognitive dysfunctions and/or with their sleep disruption.

Several methods exist to assess early changes in brain function with normal versus abnormal cognitive aging. Among them, studies that have used quantitative waking electroencephalography (EEG) showed that this approach is able to predict an abnormal cognitive decline in older adults (without considering the presence of OSA) [8]. For example, in one study performed in older adults with self-reported cognitive decline, increased theta power, slowing of the mean EEG frequency and increased intra-hemispheric EEG covariance were observed among central and posterior regions at the baseline in participants who presented an objective cognitive decline at a 7-9 years follow-up [9]. Other studies showed that mild cognitive impairment is associated with a loss of complexity in the EEG signal where the signal tends to be more regular (less complex) than for age-matched control groups [10]. In Alzheimer's disease, the same portrait 
of EEG slowing and loss of complexity was reported, but to a greater extent (see Dauwels et al., 2010 for a review [10]).

Whether young, middle-aged and older adults with OSA (without considering the cognitive decline) have changes in their waking EEG spectral power is less clear, as only a few studies have assessed EEG spectral analysis during wakeful rest in adults presenting OSA. A recent systematic review included seven studies performed in adults with OSA and showed they present a general slowing of the EEG during wakefulness and this slowing was related to markers of OSA severity (i.e., apnea-hypopnea index [AHI], oxygen desaturation and sleep fragmentation) and vigilance failure [11].

Another way to investigate brain function through waking EEG is the measurement of EEG functional connectivity (FC) described by the temporal correlation between the electrical activity of different brain regions, allowing the evaluation of the degree to which these regions interact for functional purposes [12]. In older adults with self-reported cognitive decline, FC globally decreases in the alpha and beta frequency bands (when pooling data from all electrodes to obtain a single connectivity value per frequency band) [13]. Similar but more extensive FC anomalies were found in mild cognitive impairment and Alzheimer's disease [14]. Globally, decreased FC was reported for alpha and beta frequency bands and, less frequently, in the theta band in adults with an abnormal cognitive decline, whereas both increase and decrease in FC were found in the delta band. While FC decreases have been interpreted as a consequence of the gray and white matter alterations that occur with neurodegeneration [15], increased FC has been interpreted as functional compensation preceding the decline of cognitive abilities when some subtle alterations to the white and/or gray matter begin to appear $[16,17]$. EEG FC could therefore be a promising avenue for early detection of abnormal brain function among late middle-aged and older adults 
with OSA. Yet, no study has investigated waking EEG FC in the OSA population. Interestingly, however, one study using an EEG graph-theory approach on eight participants showed that total sleep deprivation alters brain FC in a topographically specific way, with the prefrontal cortex being more affected by sleep deprivation, particularly for the alpha and theta frequency bands [18].

In the present study, we investigated whether adults aged 55-85 years old with OSA present changes in quantitative waking EEG (spectral power and FC) when compared to healthy controls. We further verified whether anomalies in spectral power and FC were related to sleep quality/quantity and neuropsychological deficits. Our hypotheses were that global EEG slowing and changes in FC typically found in older adults at risk of cognitive decline (i.e. those with selfreported cognitive decline and mild cognitive impairments) are more likely to be observed in middle-aged and older adults with OSA than in those without OSA. Moreover, because of the sleep disruption associated with OSA, we hypothesized that the frontal regions could be particularly affected.

\section{Material and methods}

\subsection{Subjects}

OSA subjects were either recruited from a hospital-based sleep apnea clinic before the beginning of their treatment or by newspapers ads. Control subjects were recruited through newspapers ads. Subjects initially enrolled as controls were switched to the OSA group when OSA was detected in polysomnography (PSG). Exclusion criteria were: dementia, neurological disorders (e.g. history of epilepsy, head injury, stroke), psychiatric conditions, non-controlled hypertension, noncontrolled diabetes, sleep disorders other than OSA, body mass index $>40$, use of medications altering vigilance or sleep (antidepressants, benzodiazepines, psychostimulants, hypnotics) and 
non-usual short total sleep time during the PSG recording $(<4 \mathrm{~h}$ while having reported an usual total sleep time $>6 \mathrm{~h}$ on the Pittsburgh Sleep Quality Index). The research protocol was approved by the Ethics' Committee of the Centre integré universitaire de santé et de services sociaux du Nord de l'île-de-Montréal. Each participant gave written informed consent before starting the study according to the declaration of Helsinki.

\subsection{Data Acquisition}

Part of the protocol was previously described in details $[19,20]$. Briefly, all subjects were evaluated with one night of in-laboratory PSG to document OSA. Upon their arrival at the laboratory, the subjects filled out the Epworth Sleepiness Scale [21], the Pittsburgh Sleep Quality Index [22], the Beck Anxiety Inventory [23] and the Beck Depression Inventory (second edition) [24]. The PSG included 17 scalp electrodes (F3, F4, F7, F8, Fz, C3, C4, Cz, T3, T4, T5, T6, P3, P4, Pz, O1 and O2) referenced to mastoids, electrooculogram, chin and anterior tibialis electromyogram, nasal and oral cannulas, a transcutaneous oximetry installed on a finger and thoracic and abdominal strain gauges. PSG recording began between 21:28 and 23:35 and ended between 6:00 and 8:07 the next morning, depending on each participant's regular sleep schedule (documented with the Pittsburgh Sleep Quality Index). Sleep stages were identified according to the American Academy of Sleep Medicine criteria [25]. Apneas were defined as an airflow reduction of $\geq 90 \%$ lasting $>$ $10 \mathrm{~s}$. Hypopneas were defined as an airflow reduction of $\geq 30 \%$ lasting $>10 \mathrm{~s}$ accompanied by either an oxygen desaturation of $\geq 3 \%$ or arousal, as recommended in 2012 [25]. Subjects with an $\mathrm{AHI} \geq 20$ were included in the OSA group while subjects with an $\mathrm{AHI} \leq 10$ were included in the control group; patients with AHI $>10$ and $<20$ were not included in the present study. These cutoffs were used to ensure the maximum difference between the control and the OSA groups while maximizing the number of subjects in each group. We recorded ten minutes of waking EEG with 
eyes closed 30 minutes after the last morning awakening, while subjects were asked to sit, relax, minimize movements and try not to fall asleep. To ensure that the subjects would not fall asleep during this procedure, we asked them to open their eyes periodically during the recording.

The morning after the PSG recording, all subjects underwent a comprehensive neuropsychological assessment. We selected tests that involved executive functions, verbal fluency and episodic memory as they are the cognitive domains most sensitive to Alzheimer's disease. The Montreal Cognitive Assessment [26], the Verbal Fluency Test (semantic and phonemic conditions) [27], the Wechsler Adult Intelligence Scale - Fourth Edition (the digit span subsection to measure the memory span) [28], the Rey-Osterrieth Complex Figure [29], the ColorWord Interference Test from the Delis-Kaplan Executive Function Scale (conditions 1 to 4) [30] and the Trail Making Test (parts A and B) [31] were used.

\subsection{Data Analysis - EEG Spectral Analysis}

We selected 4-s EEG epochs without artefact for a minimum of 100 s per subject. EEG epochs were then imported in the Brainstorm software [32]. We applied the Signal-Space Projection correction to the cardiac artefacts (standard correction available in Brainstorm). EEG power spectra were determined for each epoch using Fast Fourier Transform at a resolution of $0.25 \mathrm{~Hz}$ with Hanning taper and a 75\% window overlap ratio, as performed in previous studies [33]. Five frequency bands were analyzed: delta $(0.5-3.75 \mathrm{~Hz})$, theta $(4.00-7.75 \mathrm{~Hz})$, alpha $(8.00-12.75 \mathrm{~Hz})$, beta $1(13.00-21.75 \mathrm{~Hz})$ and beta $2(22.00-31.75 \mathrm{~Hz})$. We applied a logarithmic correction to normalize the spectral power values and replaced the values from channels with muscular artefacts with estimated values using the Fisher-Yates procedure with 200 iterations for convergence [34]. The replacement was completed with $15.7 \%$ missing data for OSA subjects, which slightly exceeds the proposed limit of $10 \%$ for better results integrity. It was however necessary to complete this 
procedure; otherwise multiple participants with EEG artefacts would have had to be excluded to ensure results integrity, and this would have altered statistical power and reliability. We then calculated the mean absolute spectral power (voltage) and relative spectral power (percentage of absolute power in a given frequency band when compared to the whole spectrum) for each frequency band, as previously described [35]. For both the absolute and the relative powers, we further averaged the power values to obtain a single mean value per region: frontal (F3, F4, F7, and F8), central (C3, C4), parietal (P3, P4), temporal (T3, T4, T5, and T6) and occipital (O1, O2). A ratio of slow (delta + theta) to fast (alpha + beta $1+$ beta 2$)$ frequencies was calculated to evaluate cortical slowing [19,33].

\subsection{Data Analysis - EEG Functional Connectivity}

EEG functional connectivity addresses the signal coupling between pairs of electrodes. Here, the coupling is defined as the quadratic coupling between amplitudes of oscillatory modes for any but stable delay across trials, i.e. the phase difference in the Fourier domain. The coherency in frequency is a metric well suited to quantify such a coupling. It is a complex-valued quantity, the amplitude of which accounts for a stable time delayed coupling between the two time-series. However, as previously noticed, this so-called mean square coherence can be dominated by spurious zero-lag (i.e. instantaneous) coupling originating from volume conduction and common reference [36]. In other words, coherency may detect false interactions between electrodes due to volume and surface conduction. While true brain interaction mostly occurs with small delays between signals, volume conduction is known to occur in a perfectly timed (zero-lag) manner. Therefore, it was proposed to consider the amplitude of the imaginary part of the coherency which excludes zero-lag interactions, thus isolating the part of coherency that necessarily reflects true non-zero lagged brain interaction [37]. Its value varies between zero (absence of coupling and 
synchrony) and one (perfect coupling and synchrony).

In the present study, we computed imaginary coherence metrics using the Brainstorm software for each frequency band (delta, theta, alpha, beta 1 and beta 2). We applied the following steps to EEG segments: 1) For each 4-s window, the EEG signal of each electrode was Fourier transformed; 2) imaginary coherence was computed for every electrode pair for each trial of 4-s, in each frequency band; 3) imaginary coherence was averaged for the total length of the selected recording for each subject; 4) a Fisher transform was applied to obtain a normal distribution for imaginary coherence values [38]; 5) a Fisher-Yates replacement [34] was applied for channels previously identified as presenting muscular artefacts (the same $15.7 \%$ missing data as for spectral analyses). Therefore, we obtained an imaginary coherence mean measure for each subject for all possible electrode pairs $(17$ electrodes $x 8$ possible combinations $=136)$, for each of the five frequency bands.

\subsection{Statistical Analyses}

SPSS was used for statistical analysis on EEG spectral power and demographic data. OSA and controls were compared using independent T-tests for demographic as well as clinical and PSG characteristics. We performed Group X Scalp region ANOVAs for each frequency band for absolute and relative spectral power. Moreover, we performed a Group X Scalp regions ANOVA for the ratio of slow to fast EEG frequencies.

For imaginary coherence, we assessed group differences using a Monte Carlo p-value in a nonparametric design. Coherence studies generally face methodological challenges as they often include large numbers of frequency bins and compare a large number of electrode pairs, which creates a multiple comparison problem. Moreover, parametric statistical tests of coherence differences rely on a normal distribution. The nonparametric design proposed by Maris \& al. [39] 
is adapted for coherence studies as it does not require data to be normally distributed and allows concurrent testing of multiple electrode pairs and frequency bands, therefore bypassing the multiple comparison problem. It also allows group sample sizes to be different.

In accordance with Maris et al. [39], p-values were compared for each condition to the critical alpha-level, which is calculated by randomly partitioning the subjects with their respective trials. To do so, the subjects of the two groups were first mixed into a single set and randomly partitioned into two surrogate data sets. Then, the test statistic from this random partition was calculated. These steps were repeated 10000 times to construct a histogram of the null-hypothesis test statistics. From the test statistic that was observed and this histogram, the proportion of random partitions that resulted in a larger test statistic than the observed one was calculated. If this proportion, called the Monte Carlo $\mathrm{p}$-value, is smaller than the critical alpha-level $(\mathrm{p}<0.01)$, the data in the two groups are considered significantly different. Given the different conditions, 170 statistical tests (17 electrodes x 5 frequency bands x 2 range's length) were performed.

To better understand the nature of the alterations in functional connectivity, the functional links for which the connectivity was changed in the OSA group at $\mathrm{p}<0.01$ compared to controls were correlated to PSG (AHI, mean oxygen saturation, total sleep time and sleep efficiency) and neuropsychological variables (Montreal Cognitive Assessment, Verbal Fluency Test- Semantic, and Color-Word Interference Test - condition 3) in the OSA group. These PSG and neuropsychological variables highly correlated with other similar PSG variables (e.g. number of awakenings, minimum oxygen saturation) and neuropsychological variables (e.g. Verbal Fluency Test - Phonemic, and Color-Word Interference Test - condition 1, 2 and 4) and were therefore selected for our analyses to avoid performing an exaggerated number of correlations that would have increased the chance of type II errors. 


\section{Results}

\subsection{Demographic and Clinical Characteristics}

This study was part of a larger research program where 139 subjects (with or without OSA) were tested with PSG, waking EEG and neuropsychology. Of these, one withdrew and 67 presented at least one exclusion criteria, including, including $\mathrm{AHI}$ limitations for this study (AHI $\geq 20$ and $\mathrm{AHI}$ $\leq 10$ respectively). We further excluded 16 OSA and 14 control subjects due to poor EEG recording quality (presence of muscular activity or eye movement artefacts or less than 100 available seconds of clean recording). A total of 19 OSA and 22 control subjects aged between 55 and 82 years old were therefore included in this study. These subjects did not differ from the 30 subjects excluded due to poor EEG recording quality on any sociodemographic variable. An average of $29.1 \pm 2.34$-s epochs of clean EEG were selected per subject, for an average length of clean EEG recording of $116.4 \pm 9$ seconds. Of the included subjects, six OSA and seven controls were diagnosed with mild cognitive impairment after our neuropsychological assessment, based on previously described criteria [40]. All these subjects were included in previous studies by our group $[19,20,41,42]$. Table 1 presents group sociodemographic and clinical characteristics. No group differences were found for age, sex, education and subjective sleepiness. OSA subjects reported poorer sleep than controls according to the Pittsburgh Sleep Quality Index. As expected, they had lower oxygen saturation, higher microarousal index, more sleep stage transitions and spent more time in stage N1 sleep compared to controls. Even though subjective sleepiness reported by the Epworth Sleepiness Scale is higher in the OSA group, this difference was not statistically different. The groups differed in none of the neuropsychological tests. 


\subsection{EEG Spectral Analysis}

When 'Group x Scalp region' ANOVAs were performed on the absolute and the relative spectral power for each frequency band separately, no significant interaction or group effect was found. Similarly, when a "Group X Scalp region" ANOVA was performed on the ratio of slow to fast EEG frequencies, no interaction or group effect was observed. As expected, multiple scalp region effects were found. These effects will not be further detailed, since it is normal that spectral power differs between brain regions, which does not relate to an OSA effect.

\subsection{EEG Functional Connectivity}

Results with imaginary coherence revealed significant differences in EEG functional connectivity networks between the two groups in four of the five frequency bands. These differences between OSA and controls for probability values of $\mathrm{p}<0.01$ are presented in Figure 1. Globally, OSA participants showed increased connectivity for nine pairs of electrodes in three different frequency bands, namely delta, theta and beta 1 . These increases in connectivity involved mostly local frontal networks as well as long-range fronto-parietal networks. More specifically, the F7-F8, F7-P3, F8P3 and F7-O1 pairs in the delta band, the C4-T5 pair in the theta band and the F8-F4, F8-Fz, F7Pz and C3-O1 pairs in the beta 1 band showed increased connectivity in OSA compared to controls. We also found reduced connectivity for four pairs of electrodes in OSA participants compared to control subjects for theta, alpha and beta 1 . These reductions in connectivity mostly involved fronto-temporal regions. More specifically, the F4-T4 and Fz-T4 pairs in the theta band, the F8T3 pair in the alpha band and the C4-T6 pair in the beta 1 band showed decreased connectivity. 


\subsection{Polysomnographic, Neuropsychological and Sleepiness Correlates of Functional}

\section{Connectivity}

We found significant correlations between PSG variables and imaginary coherence in the OSA group only (See Table 2 for statistics). For electrode pairs that showed increased connectivity in OSA compared to control participants, we found that increased connectivity correlated with reduced sleep efficiency (delta: F7-F8, F7-P3; beta 1: C3-O1) and reduced total sleep time (delta: F7-F8; beta 1: C3-O1). In addition, we found that decreased connectivity correlated with reduced sleep efficiency (theta: T4-Fz) and increased AHI (theta: F4-T4). No significant correlation was found between functional connectivity and neuropsychological variables in the OSA group.

\section{Discussion}

This study aimed at verifying whether middle-aged and older adults with untreated OSA showed differences in EEG spectral power and functional connectivity during wakefulness. Our OSA participants showed no changes in EEG spectral power when tested with a conventional statistical approach. They however presented several local and long-range functional connectivity changes mainly between frontal, temporal and parietal regions. In fact, we observed increased connectivity in the delta, theta and beta-1 bands in OSA participants in frontal and fronto-parietal regions compared to controls. These increases were associated with reduced sleep efficiency and decreased total sleep time. We also found that OSA participants have decreased EEG connectivity in the theta, alpha and beta-1 bands in fronto-temporal regions. These decreases in functional connectivity were correlated with reduced sleep efficiency and increased AHI in the night preceding the waking EEG. However, none of the functional connectivity patterns correlated with scores on neuropsychological tests. 


\subsection{Relationship between OSA and brain health}

Multiple physiological processes including oxidative stress, inflammation, hypertension, dysautonomia, impaired glucose metabolism and blood-brain barrier dysfunction may alter brain health in OSA (see Gosselin et al., 2018 for a review [2]). Not surprisingly, white matter alterations, mainly reduced fiber integrity in vulnerable regions such as the limbic system, basal ganglia, cerebellar and part of the cerebral cortex, as well as changes in gray matter structure were found in OSA $[43,44]$. These alterations in brain structure may, with time, increase the risk of cognitive decline and mimic some of the alterations seen in Alzheimer's disease $[45,46]$. Interestingly, white and gray matter alterations as well as beta-amyloid $(\mathrm{A} \beta)$ deposition were previously related to decreased functional connectivity $[15,47]$.

In our sample of participants, however, no cognitive decline was documented in the OSA group compared to the control group using neuropsychological tests. Moreover, we found gray matter hypertrophy as well as increases in cortical thickness in frontal, parietal and cingulate regions and in the amygdala in a study using the current sample [42]. We concluded that this is probably resulting from cerebral edema and changes in cellular size or quantity due to hypoxemia. We also hypothesized that participants were in a pre-symptomatic stage of the OSA disease process characterized by reactive and adaptive cerebral mechanisms rather than neuronal death and cognitive decline. Interestingly, in the present study, the increased FC between frontal and parietal areas is consistent with regions of gray matter hypertrophy and cortical thickness we previously found in our sample. Whether these changes in FC will further evolve into cognitive decline needs to be investigated in a longitudinal study.

While we mostly found FC increases between the frontal and parietal regions, we also found decreased connectivity between frontal and temporal regions in our OSA group, which was 
related to increased AHI. Similar results of simultaneous increases and decreases in FC were previously found in OSA (see Khazaie, 2017 for a review [48]). Notably, in Park et al. (2016) [49], widespread increases (in 64 functional connections) and decreases (in 49 functional connections) in cortical FC were found. Some authors interpreted increases in FC as mechanisms of brain functional compensation to prevent cognitive dysfunction, in which unaltered regions of the brain palliate for deficits observed elsewhere [16,17]. We cannot exclude the possibility of such compensatory mechanisms in our sample; this would be in line with recent theories and evidence about functional compensation in the early stages of neurodegenerative conditions $[50,51]$ and would explain why we did not find group differences in neuropsychological deficits despite decreased connectivity in fronto-temporal areas in OSA. However, increased connectivity must be interpreted with caution in a non-task related context, as the brain activity is not focused on a single objective and may therefore be influenced by other uncontrolled cerebral mechanisms such as having specific personal thoughts or initiating a movement [52]. Moreover, this increased connectivity could also reflect an attempt to fight sleepiness in the OSA group.

\subsection{Relationships between sleep and waking EEG functional connectivity}

In non-OSA individuals, sleep deprivation was associated with multiple FC changes in waking EEG and functional magnetic resonance imaging [53-58]. Notably, an EEG study evaluating the effects of a 36-h sleep deprivation revealed widespread cortico-cortical functional connectivity increases that were proportional to the number of hours spent awake [53]. In functional magnetic resonance imaging, previous studies [54-56] found decreased FC in sleep deprivation (mainly in the default mode network), but more recent studies mainly found increased FC in multiple intraand inter-hemispheric cortical regions including those of the default mode network and the sensorimotor network [57,58]. These studies showing increased FC used innovative methods such 
as the voxel-mirrored homotopic connectivity or the dynamic functional connectivity as well as extensive analyses such as testing effects with or without whole brain signal regression. Such increases were hypothesized as the compensatory involvement of brain areas to reduce cognitive performance deterioration while under high sleep pressure [58]. Considering these results and the relationship between imaginary coherence, sleep efficiency and total sleep time, functional connectivity changes could be partly due to sleep architecture alterations in our OSA group.

\subsection{Spectral analysis}

In the present study, no changes were found in OSA EEG spectral composition. These results diverge from most of those reported in a recent literature review in which five out of seven studies found higher absolute and relative EEG power in slow frequency bands (delta and theta) during wakefulness, while four studies reported a higher EEG cortical slowing ratio ((delta+theta)/(alpha+beta) absolute power) [11]. Here, we used the same methodology as in Mathieu et al. (2007) [33], one of the studies reported in D'Rozario et al. (2017) [11]. In Mathieu et al. (2007), OSA patients had higher cortical slowing ratios in all cortical regions when compared to controls. The participants, however, differed from our sample. In fact, they had more severe OSA, with mean AHI of 46.9 in the younger and 42.8 in the older group (compared to 35.6 in the present study). Their subjects also had more oxygen desaturation and more arousals than our subjects. This may explain why we did not find statistically significant differences in spectral analysis in our sample. This also further supports the possibility of a pre-symptomatic stage of the OSA disease process in our sample.

\subsection{Strengths and limitations}

The non-parametric procedure we used for EEG connectivity bypasses the multiple comparisons problem and thus offers the possibility to investigate connectivity in all available pairs of 
electrodes. We therefore obtained a complete portrait of EEG connectivity in OSA individuals. Also, imaginary coherence is a very conservative connectivity metric, which contributes to the reliability of our results. Using this metric, our results are representative of true brain interactions and exclude the possibility of connectivity overestimation due to EEG volume conduction and subsequent zero-lag signal influence [36]. However, given that it is strict, imaginary coherence is likely to miss at least parts of the brain interactions [36].

The restricted spatial resolution of the EEG limited the interpretability of functional connectivity changes between specific cortical regions. However, the use of EEG for assessment of cerebral functioning comes with a high temporal resolution and was demonstrated useful for the detection of subtle functional patterns, enabling the early detection of neurodegenerative brain dysfunctions [59].

To obtain reliable results, we had to exclude subjects with five or more channels affected by artefacts or with less than 100 seconds of clean EEG data. Since subjects with EEG artefacts may be those with difficulties to stay awake during the recording, excluding them could have reduced the significance of our results. Conversely, to include as many patients as possible in the analyses, we included subjects with up to five EEG channels affected by muscular artefacts, removed the data from the affected channels and estimated these values using the Fisher-Yates replacement. We also applied a correction for cardiac artefacts to further prevent the overestimation of connectivity values in OSA patients.

Finally, we used a criterion of $\mathrm{AHI} \leq 10$ for our control group, but this definition includes not only non-apneic (AHI < 5), but also mild OSA (typically AHI between 5 and 15). When using standard criteria of AHI < 5, up to $90 \%$ of men and $78 \%$ of women are diagnosed with OSA [1]. In our sample of 139 participants, only a few participants met the AHI < 5 criterion. We therefore 
decided to use $\mathrm{AHI} \leq 10$ for our "control" participants and AHI $\geq 20$ for our OSA group. Our two groups were statistically different for AHI (OSA: $35.6 \pm 12.6$ and Control: $4.0 \pm 2.8, \mathrm{p}<0.001)$, as well as for all sleep fragmentation and oxygen saturation variables.

\section{Conclusions}

In our sample of middle-aged and older individuals with OSA, we were able to detect changes in EEG functional connectivity that were associated with sleep efficiency and total sleep time, but not with cognitive decline. This study confirms that our non-parametric imaginary coherence method was sensitive enough to detect subtle changes in brain functioning. Increased imaginary coherence was mostly found between frontal and parietal regions, while decreased imaginary coherence was mostly found between frontal and temporal regions. These changes were independent of neuropsychological deficits, suggesting they are related to other mechanisms such as sleep alterations. Whether OSA treatment could reduce or reverse this abnormal brain functioning will need to be tested. Moreover, we will need to evaluate whether individuals who had the greatest changes in FC are more at risk of cognitive decline if not treated.

\section{Acknowledgments}

We would like to thank Jean Paquet for his help with the statistical analyses. We also thank Caroline d'Aragon, Hélène Blais, Benoît Adam, Nancy Poirier, Mélina-Crécia Babala, JeanClaude Aubertin, Joëlle Robert, Sarah-Hélène Julien and Maria Tuineag for the recruitment of subjects and EEG data acquisition, as well as Michaël Fortin for the production of the connectivity figures. 


\section{Funding}

Mr. Fortin reports other from Conseil de recherches en sciences naturelles et en génie du Canada; Dr. Desjardins reports other from Fonds de la recherche du Québec, nature et technologies; Dr. Gagnon reports personal fees from Hôpital du Sacré-Cœur de Montréal, personal fees from Fonds de la recherche du Québec - Nature et technologie; Dr. Baril reports other from Fonds de la recherche du Québec - Santé, other from Canadian Institutes of Health Research; Dr. Carrier reports grants from Canopy Health, grants from Merck, grants from Rana, grants from PhilippsRespironics, outside the submitted work, during the conduct of the study. Dr. Gosselin reports a grant and a salary award from the Fonds de la recherche du Québec - Santé and a grant from the Canadian Institutes of Health Research. 


\section{References}

[1] Senaratna CV, Perret JL, Lodge C, Lowe A, Campbell BE, Matheson MC, et al. Prevalence of Obstructive Sleep Apnea in the general population: A systematic review. Sleep Medicine Reviews 2016:1-42. doi:10.1016/j.smrv.2016.07.002.

[2] Gosselin N, Baril A-A, Osorio RS, Kaminska M, Carrier J. Obstructive Sleep Apnea and the Risk of Cognitive Decline in Older Adults. American Journal of Respiratory and Critical Care Medicine 2018:rccm.201801-0204PP-23. doi:10.1164/rccm.2018010204PP.

[3] Olaithe M, Bucks RS. Executive Dysfunction in OSA Before and After Treatment: A Meta-Analysis. Sleep 2013;36:1297-305. doi:10.5665/sleep.2950.

[4] Kilpinen R, Saunamäki T, Jehkonen M. Information processing speed in obstructive sleep apnea syndrome: a review. Acta Neurologica Scandinavica 2013;129:209-18. doi:10.1111/ane.12211.

[5] Wallace A, Bucks RS. Memory and obstructive sleep apnea: a meta-analysis. Sleep 2013;36:203-20. doi:10.5665/sleep.2374.

[6] Bucks RS, Olaithe M, Eastwood P. Neurocognitive function in obstructive sleep apnoea: a meta-review. Respirology 2013;18:61-70. doi:10.1111/j.1440-1843.2012.02255.x.

[7] Leng Y, McEvoy CT, Allen IE, Yaffe K. Association of Sleep-Disordered Breathing With Cognitive Function and Risk of Cognitive Impairment: A Systematic Review and Meta-analysis. JAMA Neurol 2017;74:1237-45. doi:10.1001/jamaneurol.2017.2180.

[8] Horvath A, Szucs A, Csukly G, Sakovics A, Stefanics G, Kamondi A. EEG and ERP biomarkers of Alzheimer's disease: a critical review. Front Biosci (Landmark Ed) 2018;23:183-220.

[9] Prichep LS, John ER, Ferris SH, Rausch L, Fang Z, Cancro R, et al. Prediction of longitudinal cognitive decline in normal elderly with subjective complaints using electrophysiological imaging. Neurobiol Aging 2006;27:471-81.

doi:10.1016/j.neurobiolaging.2005.07.021.

[10] Dauwels J, Vialatte F, Cichocki A. Diagnosis of Alzheimers Disease from EEG Signals: Where Are We Standing? Car 2010;7:487-505. doi:10.2174/156720510792231720.

[11] D'Rozario AL, Cross NE, Vakulin A, Bartlett DJ, Wong KKH, Wang D, et al. Quantitative Electroencephalogram Measures in Adult Obstructive Sleep Apnea Potential Biomarkers of Neurobehavioural Functioning. Sleep Medicine Reviews 2017:1-44. doi:10.1016/j.smrv.2016.10.003.

[12] Fingelkurts AA, Fingelkurts AA, Kähkönen S. Functional connectivity in the brain--is it an elusive concept? Neuroscience \& Biobehavioral Reviews 2005;28:827-36. doi:10.1016/j.neubiorev.2004.10.009.

[13] Koenig T, Prichep L, Dierks T, Hubl D, Wahlund L-O, John ER, et al. Decreased EEG synchronization in Alzheimer's disease and mild cognitive impairment. Neurobiol Aging 2005;26:165-71. doi:10.1016/j.neurobiolaging.2004.03.008.

[14] Babiloni C, Lizio R, Marzano N, Capotosto P, Soricelli A, Triggiani AI, et al. Brain neural synchronization and functional coupling in Alzheimer's disease as revealed by resting state EEG rhythms. Int J Psychophysiol 2016;103:88-102. doi:10.1016/j.ijpsycho.2015.02.008. 
[15] Damoiseaux JS, Greicius MD. Greater than the sum of its parts: a review of studies combining structural connectivity and resting-state functional connectivity. Brain Struct Funct 2009;213:525-33. doi:10.1007/s00429-009-0208-6.

[16] Zhang Q, Wang D, Qin W, Li Q, Chen B, Zhang Y, et al. Altered resting-state brain activity in obstructive sleep apnea. Sleep 2013;36:651-659B. doi:10.5665/sleep.2620.

[17] Park B, Palomares JA, Woo MA, Kang DW, Macey PM, Yan-Go FL, et al. Disrupted functional brain network organization in patients with obstructive sleep apnea. Brain Behav 2016;6:n/a-n/a. doi:10.1002/brb3.441.

[18] Verweij IM, Romeijn N, Smit DJ, Piantoni G, Van Someren EJ, Van Der Werf YD. Sleep deprivation leads to a loss of functional connectivity in frontal brain regions 2014;15:1-10. doi:10.1186/1471-2202-15-88.

[19] Baril A-A, Gagnon K, Gagnon J-F, Montplaisir J, Gosselin N. Association between waking electroencephalography and cognitive event-related potentials in patients with obstructive sleep apnea. Sleep Medicine 2013;14:685-7.

doi:10.1016/j.sleep.2013.03.014.

[20] Gosselin N, De Beaumont L, Gagnon K, Baril A-A, Mongrain V, Blais H, et al. BDNF Val66Met Polymorphism Interacts with Sleep Consolidation to Predict Ability to Create New Declarative Memories. J Neurosci 2016;36:8390-8. doi:10.1523/JNEUROSCI.4432-15.2016.

[21] Johns MW. A new method for measuring daytime sleepiness: the Epworth sleepiness scale. Sleep 1991;14:540-5.

[22] Buysse DJ, Reynolds CF, Monk TH, Berman SR, Kupfer DJ. The Pittsburgh sleep quality index: A new instrument for psychiatric practice and research. Psychiatry Res 1989;28:193-213.

[23] Beck AT, Epstein N, Brown G, Steer RA. An inventory for measuring clinical anxiety: psychometric properties. Journal of Consulting and Clinical Psychology 1988;56:893-7.

[24] Beck AT, Steer RA, Brown GK. Beck depression inventory-II. San Antonio 1996;78:490-8.

[25] Berry RB, Budhiraja R, Gottlieb DJ, Gozal D, Iber C, Kapur VK, et al. Rules for scoring respiratory events in sleep: update of the 2007 AASM Manual for the Scoring of Sleep and Associated Events. Deliberations of the Sleep Apnea Definitions Task Force of the American Academy of Sleep Medicine. J Clin Sleep Med 2012;8:597-619. doi:10.5664/jcsm.2172.

[26] Nasreddine ZS, Phillips NA, Bédirian V, Charbonneau S, Whitehead V, Collin I, et al. The Montreal Cognitive Assessment, MoCA: a brief screening tool for mild cognitive impairment. Journal of the American Geriatrics Society 2005;53:695-9. doi:10.1111/j.1532-5415.2005.53221.x.

[27] Lucas JA, Ivnik RJ, Smith GE, Bohac DL, Tangalos EG, Graff-Radford NR, et al. Mayo's Older Americans Normative Studies: Category Fluency Norms. Journal of Clinical and Experimental Neuropsychology 2010;20:194-200.

doi:10.1076/jcen.20.2.194.1173.

[28] Wechsler D. Wechsler adult intelligence scale-Fourth Edition (WAIS-IV). San Antonio, TX: NCS Pearson 2008;22:498.

[29] Osterrieth PA. Le test de copie d'une figure complexe. Archiv fur Psychologie 30,206356,-Corwin J, Bylsma FW (translated 1993). The Clinical Neuropsychologist 1944;7:915 . 
[30] Delis DC, Kaplan E, Kramer JH. Executive function system: examiner's manual. The Psychological Corporation, San Antonio 2001:91-103.

[31] Raitan RM. Trail Making Test. Manual for administration, scoring and interpolation 1956.

[32] Tadel F, Baillet S, Mosher JC, Pantazis D, Leahy RM. Brainstorm: A User-Friendly Application for MEG/EEG Analysis. Comp Int and Neurosc (CIN) 20112011. doi:10.1155/2011/879716.

[33] Mathieu A, Mazza S, Petit D, Décary A, Massicotte-Marquez J, Malo J, et al. Does age worsen EEG slowing and attention deficits in obstructive sleep apnea syndrome? Clinical Neurophysiology 2007;118:1538-44. doi:10.1016/j.clinph.2007.04.009.

[34] Gosselin N, Lassonde M, Petit D, Leclerc S, Mongrain V, Collie A, et al. Sleep following sport-related concussions. Sleep Medicine 2009;10:35-46. doi:10.1016/j.sleep.2007.11.023.

[35] Sleep following sport-related concussions 2009;10:35-46. doi:10.1016/j.sleep.2007.11.023.

[36] Nolte G, Bai O, Wheaton L, Mari Z, Vorbach S, Hallett M. Identifying true brain interaction from EEG data using the imaginary part of coherency. Clinical Neurophysiology 2004;115:2292-307. doi:10.1016/j.clinph.2004.04.029.

[37] Stinstra JG, Peters MJ. The volume conductor may act as a temporal filter on the ECG and EEG. Med Biol Eng Comput 1998;36:711-6.

[38] Fisher RA. Frequency Distribution of the Values of the Correlation Coefficient in Samples from an Indefinitely Large Population. Biometrika 1915;10:507. doi:10.2307/2331838.

[39] Maris E, Schoffelen J-M, Fries P. Nonparametric statistical testing of coherence differences. J Neurosci Methods 2007;163:161-75. doi:10.1016/j.jneumeth.2007.02.011.

[40] Gagnon K, Baril A-A, Montplaisir J, Carrier J, De Beaumont L, D'Aragon C, et al. Disconnection Between Self-Reported and Objective Cognitive Impairment in Obstructive Sleep Apnea. Journal of Clinical Sleep Medicine 2019;15:409-15. doi:10.5664/jcsm.7664.

[41] Gagnon K, Baril A-A, Gagnon J-F, Fortin M, Décary A, Lafond C, et al. Cognitive impairment in obstructive sleep apnea. Pathol Biol 2014;62:233-40. doi:10.1016/j.patbio.2014.05.015.

[42] Baril A-A, Gagnon K, Brayet P, Montplaisir J, De Beaumont L, Carrier J, et al. Gray Matter Hypertrophy and Thickening with Obstructive Sleep Apnea in Middle-aged and Older Adults. American Journal of Respiratory and Critical Care Medicine 2017. doi:10.1164/rcem.201606-12710C.

[43] Weng H-H, Tsai Y-H, Chen C-F, Lin Y-C, Yang C-T, Tsai Y-H, et al. Mapping gray matter reductions in obstructive sleep apnea: an activation likelihood estimation metaanalysis. Sleep 2014;37:167-75. doi:10.5665/sleep.3330.

[44] Ho B-L, Tseng P-T, Lai C-L, Wu M-N, Tsai M-J, Hsieh C-F, et al. Obstructive sleep apnea and cerebral white matter change: a systematic review and meta-analysis. J Neurol 2018;265:1643-53. doi:10.1007/s00415-018-8895-7.

[45] Palmqvist S, Schöll M, Strandberg O, Mattsson N, Stomrud E, Zetterberg H, et al. Earliest accumulation of $\beta$-amyloid occurs within the default-mode network and concurrently affects brain connectivity. Nature Communications 2017:1-13. doi:10.1038/s41467-017-01150-x. 
[46] Palmqvist S, Schöll M, Strandberg O, Mattsson N, Stomrud E, Zetterberg H, et al. Earliest accumulation of $\beta$-amyloid occurs within the default-mode network and concurrently affects brain connectivity. Nature Communications 2017:1-13. doi:10.1038/s41467-017-01150-x.

[47] Khazaie H, Veronese M, Noori K, Emamian F, Zarei M, Ashkan K, et al. Functional Reorganization in Obstructive Sleep Apnoea and Insomnia: A Systematic Review of the Resting-State fMRI. Neuroscience \& Biobehavioral Reviews 2017:1-34. doi:10.1016/j.neubiorev.2017.03.013.

[48] Barulli D, Stern Y. Efficiency, capacity, compensation, maintenance, plasticity: emerging concepts in cognitive reserve. Trends Cogn Sci (Regul Ed) 2013;17:502-9. doi:10.1016/j.tics.2013.08.012.

[49] Scheller E, Minkova L, Leitner M, Klöppel S. Attempted and successful compensation in preclinical and early manifest neurodegeneration - a review of task FMRI studies. Front Psychiatry 2014;5:132. doi:10.3389/fpsyt.2014.00132.

[50] Grady C. The cognitive neuroscience of ageing. Nat Rev Neurosci 2012;13:491-505. doi:10.1038/nrn3256.

[51] Kar S, Routray A. Effect of sleep deprivation on functional connectivity of EEG channels. IEEE Transactions on Systems 2013. doi:10.1109/TSMCA.2012.2207103.

[52] Sämann PG, Tully C, Spoormaker VI, Wetter TC, Holsboer F, Wehrle R, et al. Increased sleep pressure reduces resting state functional connectivity. Magn Reson Mater Phy 2010;23:375-89. doi:10.1007/s10334-010-0213-z.

[53] De Havas JA, Parimal S, Soon CS, Chee MWL. Sleep deprivation reduces default mode network connectivity and anti-correlation during rest and task performance. NeuroImage 2012;59:1745-51. doi:10.1016/j.neuroimage.2011.08.026.

[54] Bosch OG, Rihm JS, Scheidegger M, Landolt H-P, Stämpfli P, Brakowski J, et al. Sleep deprivation increases dorsal nexus connectivity to the dorsolateral prefrontal cortex in humans. Proceedings of the National Academy of Science 2013;110:19597-602. doi:10.1073/pnas.1317010110.

[55] Xu H, Shen H, Wang L, Zhong Q, Lei Y, Yang L, et al. Impact of $36 \mathrm{~h}$ of total sleep deprivation on resting-state dynamic functional connectivity. Brain Research 2018;1688:22-32. doi:10.1016/j.brainres.2017.11.011.

[56] Zhu Y, Feng Z, Xu J, Fu C, Sun J, Yang X, et al. Increased interhemispheric restingstate functional connectivity after sleep deprivation: a resting-state fMRI study. Brain Imaging and Behavior 2016;10:911-9. doi:10.1007/s11682-015-9490-5.

[57] Smailovic U, Koenig T, Kåreholt I, Andersson T, Kramberger MG, Winblad B, et al. Quantitative EEG Power and Synchronization correlate with Alzheimer's disease CSF biomarkers. Neurobiol Aging 2017:1-33. doi:10.1016/j.neurobiolaging.2017.11.005. 


\section{Tables}

Table 1

Demographic, mood and sleep, polysomnographic and neuropsychological characteristics

Variables $\quad$ OSA $\quad$ Controls tvalues $p$ values

$\mathrm{n}=19 \quad \mathrm{n}=22$

\section{Demographic}

Age (years)

$\operatorname{Sex}(\mathrm{N})$

Education (years)

Body Mass Index $\left(\mathrm{kg} / \mathrm{m}^{2}\right)$

$63.6 \pm 6.4 \quad 63.6 \pm 6.7 \quad-0.002 \quad .998$

$16 \mathrm{M}: 3 \mathrm{~F} \quad 15 \mathrm{M}: 7 \mathrm{~F} \quad \chi^{2}: 1.4 \quad .190$

$\begin{array}{llll}15.3 \pm 4.3 & 15.2 \pm 3.2 & 0.07 & .945\end{array}$

$30.7 \pm 3.7 \quad 26.4 \pm 3.6 \quad 3.8 \quad .001^{* * *}$

Mood and sleep questionnaires

Beck Depression Inventory

Beck Anxiety Inventory

Epworth Sleepiness Scale

Pittsburgh Sleep Quality Index

$\begin{array}{llll}8.4 \pm 5.7 & 5.6 \pm 4.8 & 1.8 & .078 \\ 4.9 \pm 4.5 & 4.2 \pm 4.7 & 0.2 & .818 \\ 9.5 \pm 4.5 & 6.7 \pm 4.6 & 2.0 & .05^{*} \\ 6.6 \pm 4.0 & 3.6 \pm 2.4 & 2.9 & .006^{* *}\end{array}$

\section{Polysomnographic}

Apnea Hypopnea Index

Mean $\mathrm{O}_{2}$ saturation

Minimum $\mathrm{O}_{2}$ saturation

Time with $\mathrm{O}_{2}$ saturation

$$
<90 \% \text { (min.) }
$$

Total sleep time (min.)

Sleep latency (min.)

Sleep efficiency $(\%)$

Sleep stage $1(\%)$

Sleep stage $2(\%)$

Sleep stage $3(\%)$

Rapid Eye Movement sleep (\%)

Microarousal index

Number of sleep stage transitions

$\begin{array}{llll}35.6 \pm 12.6 & 4.0 \pm 2.8 & 11.5 & <.001^{* * * *} \\ 94.0 \pm 1.0 & 95.0 \pm 0.8 & -3.3 & .002^{* *} \\ 81.0 \pm 8.1 & 88.9 \pm 3.1 & -4.3 & .001^{* * *} \\ 16.6 \pm 20.7 & 0.4 \pm 0.8 & 3.7 & .001^{* * *}\end{array}$

$376.6 \pm 56.9 \quad 380.8 \pm 45.8 \quad-0.3 \quad .793$

$\begin{array}{llll}14.4 \pm 19.4 & 15.3 \pm 14.8 & -0.2 & .867\end{array}$

$\begin{array}{llll}79.5 \pm 9.7 & 80.2 \pm 8.5 & -0.2 & .815\end{array}$

$28.3 \pm 14.4 \quad 17.4 \pm 5.1 \quad 3.3 \quad .002^{* * *}$

$52.5 \pm 12.2 \quad 57.7 \pm 7.4 \quad-1.7 \quad .104$

$\begin{array}{llll}5.4 \pm 4.2 & 8.4 \pm 8.0 & -1.5 & .152\end{array}$

$\begin{array}{llll}13.8 \pm 5.2 & 16.5 \pm 4.4 & -1.8 & .077\end{array}$

$20.9 \pm 8.3 \quad 12.3 \pm 5.1 \quad 4.0<.001^{\text {*** }}$

$301.6 \pm 76.4 \quad 228.5 \pm 56.0 \quad 3.5 \quad .001$

\section{Neuropsychological}

Montreal Cognitive Assessment

$26.5 \pm 2.9$

$28.0 \pm 2.5$

$-1.7$

.094

$37.2 \pm 8.4$

$40.1 \pm 7.7$

$-1.1$

.269

Verbal Fluency Test - phonemic

$35.3 \pm 8.4$

$42.8 \pm 15.0$

$-1.9$

.059

$16.4 \pm 4.4$

$17.1 \pm 4.3$

$-0.5$

.650

Rey-Osterrieth Complex Figure

$47.6 \pm 8.6$

$53.4 \pm 10.8$

$-1.9$

.071

Color-Word Interference Test

(seconds)

Condition 1

$32.4 \pm 4.9$

$30.6 \pm 4.7$

1.2 
Condition 2

Condition 3

Condition 4

Trail Making Test (seconds)

Part A

Part B

$\begin{array}{llll}21.8 \pm 3.5 & 21.2 \pm 3.4 & 0.6 & .573 \\ 60.9 \pm 10.0 & 60.6 \pm 15.8 & 0.1 & .934 \\ 64.0 \pm 14.0 & 66.6 \pm 17.0 & -0.5 & .588\end{array}$

$36.5 \pm 8.6 \quad 36.0 \pm 13.7 \quad 0.1 \quad .886$

$87.3 \pm 35.4 \quad 83.0 \pm 51.3 \quad 0.3 \quad .760$

OSA: Obstructive sleep apnea. Statistically significant $p$ values are specified as follows: ${ }^{*} p<.05 ;{ }^{* *} p<.01 ;{ }^{* * *} p$ $<.001$.

${ }^{1}$ Measured with the digit span section of the Wechsler Adult Intelligence Scale - Fourth Edition. 


\section{Table 2}

Correlations between imaginary coherence and sleep in OSA subjects

\begin{tabular}{lllll}
\hline & & & $r$ values & $p$ values \\
\hline Sleep Efficiency & Delta & F7-F8 & -.55 & $.014^{*}$ \\
& & F7-P3 & -.46 & $.046^{*}$ \\
& Theta & T4-Fz & .51 & $.026^{*}$ \\
& Beta1 & C3-O1 & -.52 & $.022^{*}$ \\
Total sleep time & Delta & F7-F8 & -.51 & $.028^{*}$ \\
& Beta1 & C3-O1 & -.51 & $.026^{*}$ \\
Apnea Hypopnea Index & Theta & F4-T4 & -.51 & $.025^{*}$ \\
Mean O2 saturation & & & All ns & \\
Epworth Sleepiness Scale & Beta1 & F4-F8 & -.54 & $.018^{*}$ \\
ns: non-significant; OSA: Obstructive sleep apnea. Statistically significant $p$ values are specified \\
as follows: ${ }^{*} p<.05 ;{ }^{* *} p<.01 ;{ }^{* * *} p<.001$.
\end{tabular}




\section{Figures}

\section{Delta}

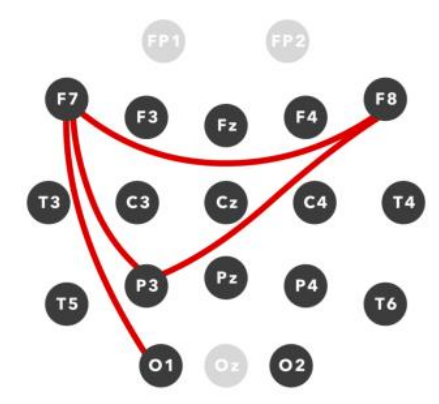

Alpha

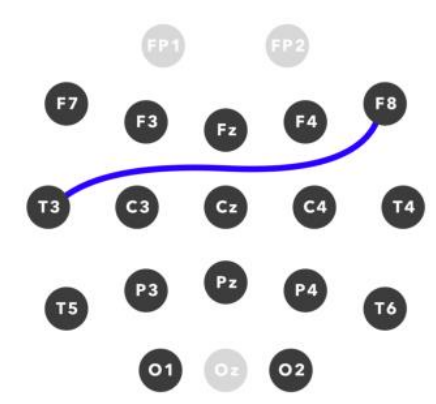

Theta

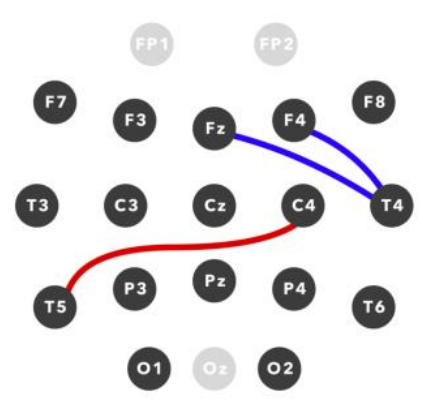

Beta 1

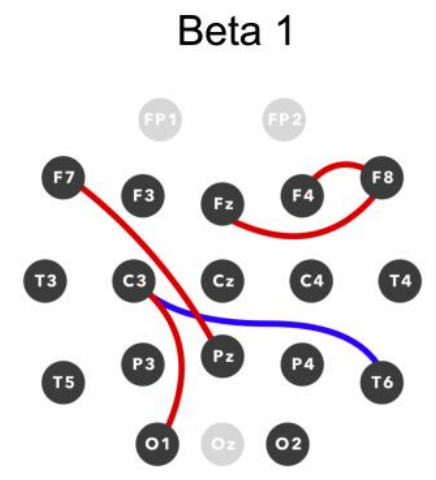

Figure 1 - Electrode pairs showing statistically significant differences between OSA and control groups in the delta, theta, alpha and beta 1 frequency bands using imaginary coherence $(p<.01)$; red links represent higher connectivity in the OSA group, blue links represent lower connectivity in the OSA group. Results in the beta 2 band are not displayed since no statistically significant connectivity difference was found within this band. Electrodes in light gray were not included in the analyses. 